\title{
TLR4 plays a crucial role in MSC-induced inhibition of NK cell function
}

\author{
$\underline{\text { Ying Lu }}^{1}$; Jin Liu ${ }^{2}$; Yang Liu $^{2}$; Yaru Qin ${ }^{2}$; Qun Luo ${ }^{1}$ Q Quanli Wang ${ }^{1 *} ;$ Haifeng Duan ${ }^{2 *}$ \\ Ying Lu and Jin Liu contribute equal to this work.
}

1 No. 307 hospital of the Chinese People's Liberation Army, Beijing, China

2 Beijing Institute of Radiation Medicine, Beijing, China

*Co-Corresponding author: Quanli Wang, No. 307 hospital of the Chinese People's Liberation Army, No.8, Dongdajie Street, Fengtai District, Beijing 100071, China.

Fax: +86-10-66947206. E-mail: 13691110351@163.com

*Co-Corresponding author: Haifeng Duan, Beijing Institute of Radiation Medicine (BIRM), No.27, Taiping Road, Haidian District, Beijing 100850, China.

Fax: +86-10-68214653. E-mail: duanhf0720@163.com 


\begin{abstract}
Mesenchymal stem cells (MSC) are a kind of stromal cell within the tumor microenvironment. In our research, MSC derived from acute myeloid leukemia patients' bone marrow (AML-MSC) and lung cancer tissues (LC-MSC) as well as normal bone marrow-derived MSC (BM-MSC) cultured in conditioned medium of HeLa cells were found to have higher expressions of Toll-like receptor (TLR4) mRNA compared with BM-MSC. The sorted TLR4-positive MSC (TLR4+ MSC) differed in cytokine (interleukin-6, interleukin-8, and monocyte chemoattractant protein-1) secretion from those of unsorted MSC. MSC was reported to inhibit natural killer (NK) cell proliferation and function. In this research, we confirmed that TLR4+ MSC aggravate this suppression. Furthermore, when TLR4 in the sorted cells were stimulated by LPS or following blocked by antibody, the suppression on NK cell proliferation and cytotoxicity were more intensive or recovered respectively. Compared to unsorted MSC, NKG2D receptor expression on NK cells were also inhibited by TLR4+ MSC. These findings suggest that activation of TLR4 pathway is important for TLR4+ MSC and MSC to obstruct anti-tumor immunity by inhibiting NK cell function, which may provide a potential stroma-targeted tumor therapy.
\end{abstract}

Key words: MSC; TLR4; NK cells; anti-tumor immunity 


\section{Introduction}

Cancer-associated fibroblasts (CAF), an important cell type in tumor stroma, is considered responsible for tumor progression by inducing angiogenesis, recruiting bone marrow-derived endothelial progenitor cells, and remodeling the extracellular matrix[1,2,3]. CAF is also expected to play a role in regulating immune cell function. Recent studies have shown that CAF from human lung cancer interact with $\mathrm{T}$ cells to enhance interferon (IFN)-c and interleukin (IL)-17A secretion[4,5].

Natural killer (NK) cells, which are part of the innate immune system, play a major role in defending a host against tumors[6]. CAF was recently reported to contribute to immunopathogenesis by inhibiting NK cell function in co-culture experiments[7]. When NK cells were co-cultured with CAF, the expressions of NK receptors (CD69, NKG2D, DNAM-1, NKp30, and NKp44) and cytolytic granzyme B were suppressed. Meanwhile, tumor necrosis factor- $\alpha$ $(\mathrm{TNF}-\alpha)$ and IFN- $\gamma$ production and cytotoxic activity were inhibited. In contrast, normal skin fibroblasts had little effect on NK cell phenotype and function[8].

Mesenchymal stem cells (MSC) are non-hematopoietic multipotent cells that may be isolated from bone marrow, adipose tissue, and some other tissues[9,10]. As a kind of stromal cell in the tumor microenvironment, MSC secrete cytokines and chemokines that contribute to tumor growth and metastasis[11,12]. MSC-derived monocyte chemotactic protein-1 (MCP-1) has been demonstrated to promote breast cancer cell migration[13,14]. MSC isolated from patients with breast cancer was demonstrated to promote MCF-7 cell proliferation and migration[15]. Also, MSC can inhibit the IL-2-induced proliferation of un-activated NK cells[16].

Both MSC and CAF are stromal cells in the tumor microenvironment, and MSC can be induced to develop into CAF-like cells. Given that both can promote tumor progression by suppressing NK cell proliferation and activity, we are eager to investigate the potential molecules that are responsible for the inhibitory effects. Interestingly, Toll-like receptor (TLR4) mRNA expression was much higher in MSC derived from different tumor tissues than that from the bone marrow of healthy volunteers. The sorted TLR4-positive MSC (TLR4+ MSC) from normal bone marrow has a stronger inhibitory effect on NK cell proliferation and cytotoxicity, indicating that

TLR4 may play an important role in MSC-mediated immunosuppression in the tumor microenvironment. 


\section{Materials and methods}

\section{Human subjects}

Three patients with leukemia, three with lung cancer, and three healthy volunteers were recruited in this study. All nine volunteers provided written informed consent.

\section{Cell culture}

The human leukemia cell line K562 and human cervical carcinoma cell line HeLa were purchased from American Type Culture Collection and maintained in RPMI 1640 medium (Gibco, NY, USA) supplemented with $10 \%$ fetal bovine serum (FBS; Hyclone, UT, USA). Cells were all incubated at $37^{\circ} \mathrm{C}$ in a humidified atmosphere containing $5 \% \mathrm{CO}_{2}$. Human MSC were isolated from the bone marrow of the healthy volunteers (BM-MSC) and patients with acute myeloid leukemia (AML-MSC) as well as from the patients' lung cancer tissues (LC-MSC). MSC from different tissues were cultured in basic $\alpha$-minimum essential medium ( $\alpha$-MEM; Gibco) supplemented with $10 \%$ FBS.

Meanwhile, bone marrow MSC were also cultured in conditioned medium of HeLa cells $\left(\mathrm{CM}^{\mathrm{HeLa}}\right)$ supplemented with $10 \%$ FBS for 2-3 weeks, which we named $\mathrm{CM}^{\mathrm{HeLa}}$-MSC. To collect conditioned medium, HeLa cells were cultured in complete Dulbecco's MEM medium. When the cell confluence was about $90 \%$, the culture medium was replaced by MSC basic $\alpha$-MEM. The $\alpha$-MEM supernatant was collected $24 \mathrm{~h}$ later and centrifuged $(1000 \mathrm{r} / \mathrm{min} \times 5 \mathrm{~min})$ for harvesting of the conditioned medium.

\section{Isolation and culture of $\mathrm{NK}$ cells}

NK cells were isolated from healthy blood using a Ficoll-Paque gradient and negative magnetic selection[17,18]. NK cell purity was $>90 \%$ as evaluated by a flow cytometry assay. Purified NK cells were cultured in RPMI 1640 supplemented with 10\% FBS containing 1000 $\mathrm{U} / \mathrm{mL}$ IL-2.

When co-cultured, 6-well polycarbonate trans-well inserts ( $8 \mu \mathrm{m}$; Corning, NY, USA) were used. BM-MSC and TLR4+ MSC were seeded in the lower compartment of the well with $5 \times 10^{4}$ cells/ well. After 6-8 h, NK cells were seeded in the upper insert with $5 \times 10^{5}$ cells/well. After co-culturing for $48 \mathrm{~h}$, NK cells were harvested for the following experiments.

\section{Immunophenotyping and flow cytometric assay}


Flow cytometric analysis was performed to identify the immunophenotype of TLR4+ MSC TLR4+ MSC $\left(1.0 \times 10^{6}\right.$ cells/sample) were stained by fluorescein isothiocyanate (FITC) -conjugated anti-human CD19 and CD34, and phycoerythrin (PE) -conjugated anti-human CD45, CD73, CD90, CD105, CD11b, and HLA-DR antibodies (Biolegend, CA, USA) for $30 \mathrm{~min}$ at room temperature. After being washed twice by phosphate buffered saline (PBS), the cells were analyzed by FACScan flow cytometry (Beckman FC500, CA, USA). TLR4+ MSC were also assayed using an allophcocyanin (APC)-conjugated anti-human TLR4 antibody (R\&D Systems, MN, USA). Similarly, NK cells were analyzed using an APC-conjugated anti human NKG2D (Biolegend) antibody following the same protocol.

\section{Adipogenic and osteogenic differentiation}

MSCs were cultured in a 24-well plate in complete $\alpha$-MEM medium supplemented with adipogenic and osteogenic-inducing agents (Sigma-Aldrich, MO, USA) at an initial cell density of $1 \times 10^{4}$ cells/well. After 2-3 weeks, cells were washed twice with PBS and fixed by $4 \%$ paraformaldehyde at room temperature for $30 \mathrm{~min}$. Oil-red-O or alkaline phosphatase staining was applied to detect adipogenic and osteogenic differentiation.

\section{Real-time quantitative polymerase chain reaction (qPCR)}

Total RNA was extracted from cells using RNA isolation kits (TRizol; Invitrogen, CA, USA). The isolated total RNA was reverse-transcribed into cDNA using qPCR Reverse Transcriptase MIX Kit (Toyobo, Osaka, Japan). The qPCR reactions were carried out using the Fast SYBR Green PCR Master Mix (Applied Biosystems Inc., CA, USA) on an ABI 7500 fast real-time PCR system (Applied Biosystems Inc.). For normalization, threshold cycles (Ct-values) were normalized to $\beta$-actin within each sample to obtain sample-specific $\Delta \mathrm{Ct}$ values $\left(\Delta \mathrm{Ct}=\mathrm{Ct}_{\mathrm{gene}}\right.$ of

interest $\left.-\mathrm{Ct}_{\beta \text {-actin }}\right)$. The values of $2^{-\Delta \Delta \mathrm{Ct}}$ were calculated to obtain fold expression levels, where $\Delta \Delta \mathrm{Ct}$ $=\left(\Delta \mathrm{Ct}_{\text {tumor-MSC }}-\Delta \mathrm{Ct}_{\mathrm{BM}-\mathrm{MSC}}\right)$.

\section{Enzyme-linked immunosorbent assay (ELISA)}

The quantitative determination of IL-6, IL-8, TNF- $\alpha$ and MCP-1 in the medium supernatant of MSC or TLR4+ MSC was tested using commercial ELISA kits (Neobioscience, Shenzhen, China) according to the manufacturer's protocols.

\section{Cell proliferation assay}

The cell proliferation assay was carried out using a cell counting kit (CCK-8; DOJINDO, 
Kumamoto, Japan). After being co-cultured with BM-MSC and TLR4+ MSC in trans-well inserts, NK cells were transferred into a 96-well plate at a density of $2 \times 10^{3}$ cells per well and $10 \mu \mathrm{L}$ of CCK-8 was added to each well and incubated at $37^{\circ} \mathrm{C}$ for $2 \mathrm{~h}$. The $\mathrm{OD}_{450}$ of all samples was detected by a microplate reader (Model 680; Bio-Rad, PA, USA).

\section{Cytotoxicity assays}

To determine NK cell cytotoxicity, the CytoTox 96 Nonradioactive Cytotoxicity Assay (Promega, WI, USA) based on the calorimetric detection of the released enzyme lactate dehydrogenase (LDH) was used. After 48-h co-culture with MSC or TLR4+ MSC, NK cells were collected and used as effectors in the cytotoxicity assays. As target cells, K562 $\left(5 \times 10^{3}\right.$ cells/well $)$ were co-cultured with NK cells $\left(5 \times 10^{4}\right.$ cells/well $)$ for $4 \mathrm{~h}$ at $37^{\circ} \mathrm{C}$ in 96 -well U-bottom plates. The assay was performed according to the manufacturer's protocol. All of the conditions were tested in quadruplicate. The absorbance of the culture medium background and lysis solution was subtracted and the percentage cytotoxicity was calculated as follows:

$$
\text { cytotoxicity }(\%)=\frac{\text { Experimental }- \text { Effector spontaneous }- \text { Target spontaneous }}{\text { Target maximum }- \text { Target spontaneous }}
$$

\section{Animal experiments}

All animals used in this study were purchased from the Laboratory Animal Center of the Academy of Military Medical Sciences (Beijing, China). The animals were treated humanely and all of the animal experimental procedures received approval from the Animal Use and Care Committee of the Beijing Institute of Radiation Medicine.

Twenty-four 6-week-old female C57BL/6J mice were randomly divided into three groups. Each group contained eight mice kept in two cages. Mice in the three groups were administered physiological saline $(0.1 \mathrm{~mL})$, MSC $\left(2 \times 10^{6}\right.$ cells suspended in $0.1 \mathrm{~mL}$ of physiological saline), and TLR4+ MSC $\left(2 \times 10^{6}\right.$ cells suspended in $0.1 \mathrm{~mL}$ physiological saline $)$ twice on day 1 and day 15 of the treatment by intravenous injection. Four weeks later, blood was collected from the retro-orbital sinus of each mouse and the peripheral blood mononuclear cells (PBMC) within it were harvested using Red Blood Cell Lysis Buffer (Beyotime, Shanghai, China) according to the manufacturer's instructions. The amounts of NK cells in the PBMC were analyzed using an anti-DX5 antibody (Biolegend) in a flow cytometric assay. 


\section{Results}

\section{Tumor-derived MSC had higher TLR4 expression}

BM-MSC, AML-MSC, LC-MSC, and $\mathrm{CM}^{\mathrm{HeLa}}$-MSC were isolated and cultured. On real-time qPCR, the relative amounts of TLR4 mRNA in the AML-MSC, LC-MSC, and CM ${ }^{\mathrm{H} L \mathrm{La}}$-MSC were significantly higher than that in BM-MSC (abbreviated as MSC in all of the figures) (Fig. 1a). This finding led us to consider the relationship between TLR4 expression in MSC and tumor development.

\section{TLR4 positive MSC maintained the same immunophenotype and differentiation ability with unsorted MSC}

To investigate the correlation between TLR4 expression in MSC and tumor development, BM-MSC were sorted and TLR4+ MSC were collected. As a precondition of sorting, the ratio of TLR4-positive cells in BM-MSC was nearly $13.3 \%$ according to the flow cytometry test result (Fig. 1c). After sorting and amplifying culture, the ratio was increased to $79.6 \%$ and the mean fluorescence, which represented the relative cellular TLR4 expression, was enhanced from 13.6 to 34.3 (Figs. 1b, 1c).

MSC was defined by a series of surface markers and multiple differentiation ability, including osteogenic and adipogenic differentiation[19,20,21,22,23]. In our research, the surface makers of TLR4+ MSC were tested by flow cytometry, revealing a coincidental immunophenotype with unsorted MSC, which was positive expression of CD90, CD73, and CD105 and negative expression of CD45, CD19, CD34, CD11b, and HLA-DR (Fig. 2a). In addition, TLR4+ MSC maintained the same ability as unsorted MSC to differentiate into osteogenic cells and adipogenic cells (Fig. 2b).

\section{Cytokine expression of TLR4+ MSC is distinguished from MSC}

ELISA was performed to detect the cytokine secretion of TLR4+ MSC. As shown in Fig. 3a, IL-6 and IL-8 were secreted at lower levels by TLR4+ MSC with significant difference compared with unsorted MSC. In contrast, MCP-1 was present at a much higher concentration in the culture supernatant of TLR4+ MSC. When MSC were stimulated by lipopolysaccharide (LPS; 100 $\mathrm{ng} / \mathrm{mL}$ ), an agonist of TLR4, the secretions of IL-6 and IL-8 were both greatly enhanced on day 1 and their concentrations were preserved at higher levels than the initial value for 4 days (Fig. 3b). 
Interestingly, the secretion enhancement of the two cytokines was much more significant in TLR4+ MSC, probably due to the enriched TLR4 expression (Fig. 3b). However, the secretions of IL-6 and IL-8 by TLR4+ MSC decreased quickly in the following 4 days, a finding that is quite different from MSC.

\section{TLR4+ MSC inhibit NK cell proliferation in vitro and in vivo}

MSC and TLR4+ MSC were seeded in 6-well plates for co-culturing with NK cells for $48 \mathrm{~h}$ using trans-well inserts. TLR4 blocking antibody (Invivogen, CA, USA) was added in one of the TLR4+ MSC-plating wells. The normally cultured and co-cultured NK cells in the inserts were then harvested to a 96-well plate for a cell counting test. As a result, the NK cell proliferation was suppressed by TLR4+ MSC. What's more, when TLR4 in the sorted cells were activated exogenously by LPS for 48h, the suppression was more remarkable. When the TLR4 function was blocked, the suppression on NK cell proliferation was recovered (Fig. 4a). Saline, MSC, and TLR4+ MSC were intravenously injected into C57BL/6J mice and PBMC were harvested from them 4 weeks later for flow cytometry assay. As shown in Fig. 4b, the percentage of NK cells in the PBMC was decreased in TLR4+ MSC-treated mice (7.3\%) compared with MSC-treated mice $(8.8 \%)$. Based on these results, TLR4+ MSC inhibit NK cell growth both in vitro and in vivo.

\section{TLR4+ MSC inhibit NK cell NKG2D expression and cytotoxicity}

MSC and TLR4+ MSC were seeded in 6-well plates for $48 \mathrm{~h}$ co-culturing with NK cells using trans-well inserts. On flow cytometry, NKG2D receptor expression on NK cells co-cultured with TLR4+ MSC was decreased to $72 \%$, whereas it was $81 \%$ and $76.6 \%$ when co-cultured with unsorted MSC or normally cultured (Fig. 4c). A cytotoxicity test was also performed. After co-culturing with MSC or TLR4+ MSC, the NK cells were collected and co-cultured with K562 cells to test for cytotoxicity using the CytoTox 96 Nonradioactive Cytotoxicity Assay. As shown in Fig. 4d, the cytotoxicity of NK cells cultured in TLR4+ MSC conditioned medium was suppressed by nearly $27 \%$ compared with that of MSC conditioned medium. When the co-cultured TLR4+ MSC were stimulated by exogenous LPS for $48 \mathrm{~h}$, the suppression of NK cell cytotoxicity was more significant. However, when the TLR4 function was blocked, the suppression on NK cell cytotoxicity was recovered. These results revealed that activation of TLR4-mediated signaling is crucial for TLR4+ MSC to inhibit NK cell function. 


\section{Discussion}

CAF, a group of stromal cells within the tumor microenvironment, has attracted attention for its pro-tumorigenic potential. CAF secretes various growth factors, including human growth factor (HGF), transforming growth factor- $\beta$ (TGF- $\beta$ ), vascular endothelial growth factor (VEGF), and NK4 [24,25,26], which result in tumor growth. Moreover, it was proven to promote tumor invasion, metastasis, and angiogenesis[27] and regulate inflammation and extracellular matrix remodeling[28,29] .

MSC, an important source of CAF, are recruited to the tumor matrix to create myofibroblasts[30]. MSC may acquire the CAF phenotype when cultured in conditioned medium of tumor cells[31,32]. MSC inhibit PBMC migration toward tumor cells and protect them against immune clearance. Meanwhile, MSC inhibited NK cell functions[33].

In our previous study, MSC derived from different tissues were isolated and cultured. Interestingly, tumor-derived MSC (AML-MSC, LC-MSC, or $\mathrm{CM}^{\mathrm{HeLa}}$-MSC) expressed higher levels of TLR4 mRNA than BM-MSC. These findings led us to consider the significance of TLR4 expression in MSC and its contribution to tumor development.

TLR4 is thought to activate the immune response to clear pathogenic microorganisms. It was reported that TLR4 activation in tumor cells or MSC may promote prostate epithelial cell proliferation[34] or trigger immune defense. In a transgenic colitis-induced colorectal cancer model, mice with LPS-TLR4 signal abrogation were markedly protected against colon carcinogenesis[35].

In our study, the sorted TLR4+ MSC more powerfully inhibited NK cell function than unsorted cells as evidenced by inhibited NK cell proliferation in vivo and in vitro. When NK cells were co-cultured with TLR4+ MSC in the transwell system, NK cell cytotoxicity was decreased by $27 \%$. Further evidently, when TLR4 in the sorted cells were stimulated by LPS or blocked by antibody, the suppression on NK cell proliferation and cytotoxicity were more intensive or recovered, suggesting that TLR4 may play an important role in the MSC-induced immunosuppression of NK cells.

On ELISA, IL-6 and IL-8 were secreted at lower levels by TLR4+ MSC than by MSC, whereas MCP-1 was secreted at a higher level. We hypothesized that cytokine regulation may affect NK receptors (CD69, NKG2D, DNAM-1, NKp30, and NKp44) or cytolytic granzyme B 
and then inhibit NK cell cytotoxicity. In our research, the NKG2D expression of NK cells was indeed reduced by TLR4+ MSC. The key molecule and mechanism of this suppression will be investigated in a future study.

Notably, the greatly enhanced secretion of IL-6 and IL-8 by LPS-stimulated TLR4+ MSC was quickly decreased in the following 4 days (Fig. 3b), which is quite different from the lasting enhancement of unsorted MSC. This result indicates that TLR4+ MSC are easily imitated by and respond intensely to a pro-inflammatory tumor microenvironment, thereby obstructing anti-tumor immunity functions such as NK cell function inhibition.

In conclusion, TLR4 contributes greatly to the MSC-induced inhibition of NK cell function. This finding may provide a new option for stroma-targeted tumor therapy.

\section{Acknowledgments}

This work was supported by the National Science Foundation of China (Nos. 81270894 and $81201760)$.

\footnotetext{
Abbreviations

MSC, mesenchymal stem cells; CAF, cancer-associated fibroblasts; NK, nature killer; TLR4, Toll-like receptor; LPS, lipopolysaccharide; TLR4+ MSC, TLR4-positive MSC; CM, conditioned medium; TNF- $\alpha$, tumor necrosis factor- $\alpha$
} 


\section{References}

[1] M. Allinen, R. Beroukhim, L. Cai, C. Brennan, J. Lahti-Domenici, H. Huang, D. Porter, M. Hu, L. Chin, A. Richardson, S. Schnitt, W.R. Sellers, K. Polyak, Molecular characterization of the tumor microenvironment in breast cancer, Cancer Cell 6 (2004) 17-32.

[2] K. Pietras, J. Pahler, G. Bergers, D. Hanahan, Functions of paracrine PDGF signaling in the proangiogenic tumor stroma revealed by pharmacological targeting, PLoS Med 5 (2008) e19.

[3] L.E. Littlepage, M. Egeblad, Z. Werb, Coevolution of cancer and stromal cellular responses, Cancer Cell 7 (2005) 499-500.

[4] X. Su, J. Ye, E.C. Hsueh, Y. Zhang, D.F. Hoft, G. Peng, Tumor microenvironments direct the recruitment and expansion of human Th17 cells, J Immunol 184 (2010) 1630-1641.

[5] J.L. Barnas, M.R. Simpson-Abelson, S.P. Brooks, R.J. Kelleher, Jr., R.B. Bankert, Reciprocal functional modulation of the activation of $\mathrm{T}$ lymphocytes and fibroblasts derived from human solid tumors, J Immunol 185 (2010) 2681-2692.

[6] A. Cerwenka, L.L. Lanier, Natural killer cells, viruses and cancer, Nat Rev Immunol 1 (2001) 41-49.

[7] T. Li, Y. Yang, X. Hua, G. Wang, W. Liu, C. Jia, Y. Tai, Q. Zhang, G. Chen, Hepatocellular carcinoma-associated fibroblasts trigger NK cell dysfunction via PGE2 and IDO, Cancer Lett 318 (2012) 154-161.

[8] T. Li, S. Yi, W. Liu, C. Jia, G. Wang, X. Hua, Y. Tai, Q. Zhang, G. Chen, Colorectal carcinoma-derived fibroblasts modulate natural killer cell phenotype and antitumor cytotoxicity, Med Oncol 30 (2013) 663.

[9] K. Bieback, S. Kern, A. Kocaomer, K. Ferlik, P. Bugert, Comparing mesenchymal stromal cells from different human tissues: bone marrow, adipose tissue and umbilical cord blood, Biomed Mater Eng 18 (2008) S71-76.

[10] C.M. Digirolamo, D. Stokes, D. Colter, D.G. Phinney, R. Class, D.J. Prockop, Propagation and senescence of human marrow stromal cells in culture: a simple colony-forming assay identifies samples with the greatest potential to propagate and differentiate, Br J Haematol 107 (1999) 275-281.

[11] N.Z. Kuhn, R.S. Tuan, Regulation of stemness and stem cell niche of mesenchymal stem cells: implications in tumorigenesis and metastasis, J Cell Physiol 222 (2010) 268-277.

[12] A.E. Karnoub, A.B. Dash, A.P. Vo, A. Sullivan, M.W. Brooks, G.W. Bell, A.L. Richardson, K. Polyak, R. Tubo, R.A. Weinberg, Mesenchymal stem cells within tumour stroma promote breast cancer metastasis, Nature 449 (2007) 557-563.

[13] R.H. Goldstein, M.R. Reagan, K. Anderson, D.L. Kaplan, M. Rosenblatt, Human bone marrow-derived MSCs can home to orthotopic breast cancer tumors and promote bone metastasis, Cancer Res 70 (2010) 10044-10050.

[14] A.P. Molloy, F.T. Martin, R.M. Dwyer, T.P. Griffin, M. Murphy, F.P. Barry, T. O'Brien, M.J. Kerin, Mesenchymal stem cell secretion of chemokines during differentiation into osteoblasts, and their potential role in mediating interactions with breast cancer cells, Int J Cancer 124 (2009) 326-332.

[15] C. Zhang, W. Zhai, Y. Xie, Q. Chen, W. Zhu, X. Sun, Mesenchymal stem cells derived from breast cancer tissue promote the proliferation and migration of the MCF-7 cell line, Oncol Lett 6 (2013) 1577-1582.

[16] G.M. Spaggiari, A. Capobianco, S. Becchetti, M.C. Mingari, L. Moretta, Mesenchymal stem 
cell-natural killer cell interactions: evidence that activated NK cells are capable of killing MSCs, whereas MSCs can inhibit IL-2-induced NK-cell proliferation, Blood 107 (2006) 1484-1490.

[17] A. Pradier, J. Passweg, J. Villard, V. Kindler, Human bone marrow stromal cells and skin fibroblasts inhibit natural killer cell proliferation and cytotoxic activity, Cell Transplant 20 (2011) 681-691.

[18] G.M. Spaggiari, A. Capobianco, H. Abdelrazik, F. Becchetti, M.C. Mingari, L. Moretta, Mesenchymal stem cells inhibit natural killer-cell proliferation, cytotoxicity, and cytokine production: role of indoleamine 2,3-dioxygenase and prostaglandin E2, Blood 111 (2008) 1327-1333.

[19] M. Dominici, K. Le Blanc, I. Mueller, I. Slaper-Cortenbach, F. Marini, D. Krause, R. Deans, A. Keating, D. Prockop, E. Horwitz, Minimal criteria for defining multipotent mesenchymal stromal cells. The International Society for Cellular Therapy position statement, Cytotherapy 8 (2006) 315-317.

[20] M.F. Pittenger, A.M. Mackay, S.C. Beck, R.K. Jaiswal, R. Douglas, J.D. Mosca, M.A. Moorman, D.W. Simonetti, S. Craig, D.R. Marshak, Multilineage potential of adult human mesenchymal stem cells, Science 284 (1999) 143-147.

[21] S.E. Haynesworth, M.A. Baber, A.I. Caplan, Cell surface antigens on human marrow-derived mesenchymal cells are detected by monoclonal antibodies, Bone 13 (1992) 69-80.

[22] A.M. Mackay, S.C. Beck, J.M. Murphy, F.P. Barry, C.O. Chichester, M.F. Pittenger, Chondrogenic differentiation of cultured human mesenchymal stem cells from marrow, Tissue Eng 4 (1998) 415-428.

[23] B. Zavan, C. Giorgi, G.P. Bagnara, V. Vindigni, G. Abatangelo, R. Cortivo, Osteogenic and chondrogenic differentiation: comparison of human and rat bone marrow mesenchymal stem cells cultured into polymeric scaffolds, Eur J Histochem 51 Suppl 1 (2007) 1-8.

[24] C. Kuperwasser, T. Chavarria, M. Wu, G. Magrane, J.W. Gray, L. Carey, A. Richardson, R.A. Weinberg, Reconstruction of functionally normal and malignant human breast tissues in mice, Proc Natl Acad Sci U S A 101 (2004) 4966-4971.

[25] C. Berking, R. Takemoto, H. Schaider, L. Showe, K. Satyamoorthy, P. Robbins, M. Herlyn, Transforming growth factor-beta1 increases survival of human melanoma through stroma remodeling, Cancer Res 61 (2001) 8306-8316.

[26] T.V. Byzova, C.K. Goldman, N. Pampori, K.A. Thomas, A. Bett, S.J. Shattil, E.F. Plow, A mechanism for modulation of cellular responses to VEGF: activation of the integrins, Mol Cell 6 (2000) 851-860.

[27] P.P. Goh, D.M. Sze, B.D. Roufogalis, Molecular and cellular regulators of cancer angiogenesis, Curr Cancer Drug Targets 7 (2007) 743-758.

[28] C. Servais, N. Erez, From sentinel cells to inflammatory culprits: cancer-associated fibroblasts in tumour-related inflammation, J Pathol 229 (2013) 198-207.

[29] C. Gaggioli, S. Hooper, C. Hidalgo-Carcedo, R. Grosse, J.F. Marshall, K. Harrington, E. Sahai, Fibroblast-led collective invasion of carcinoma cells with differing roles for RhoGTPases in leading and following cells, Nat Cell Biol 9 (2007) 1392-1400.

[30] N.C. Direkze, M.R. Alison, Bone marrow and tumour stroma: an intimate relationship, Hematol Oncol 24 (2006) 189-195.

[31] P.J. Mishra, R. Humeniuk, D.J. Medina, G. Alexe, J.P. Mesirov, S. Ganesan, J.W. Glod, D. 
Banerjee, Carcinoma-associated fibroblast-like differentiation of human mesenchymal stem cells, Cancer Res 68 (2008) 4331-4339.

[32] J. Harper, R.C. Sainson, Regulation of the anti-tumour immune response by cancer-associated fibroblasts, Semin Cancer Biol 25 (2014) 69-77.

[33] S.A. Patel, J.R. Meyer, S.J. Greco, K.E. Corcoran, M. Bryan, P. Rameshwar, Mesenchymal stem cells protect breast cancer cells through regulatory $\mathrm{T}$ cells: role of mesenchymal stem cell-derived TGF-beta, J Immunol 184 (2010) 5885-5894.

[34] S.D. Kundu, C. Lee, B.K. Billips, G.M. Habermacher, Q. Zhang, V. Liu, L.Y. Wong, D.J. Klumpp, P. Thumbikat, The toll-like receptor pathway: a novel mechanism of infection-induced carcinogenesis of prostate epithelial cells, Prostate 68 (2008) 223-229.

[35] M. Fukata, A. Chen, A.S. Vamadevan, J. Cohen, K. Breglio, S. Krishnareddy, D. Hsu, R. Xu, N. Harpaz, A.J. Dannenberg, K. Subbaramaiah, H.S. Cooper, S.H. Itzkowitz, M.T. Abreu, Toll-like receptor-4 promotes the development of colitis-associated colorectal tumors, Gastroenterology 133 (2007) 1869-1881. 


\section{Figure legend}

Figure 1. Analysis of Toll-like receptor 4 (TLR4) expression in mesenchymal stem cells (MSC) derived from different tissues and sorted TLR4+ MSC

(a) The relative amounts of TLR4 mRNA in cells derived from patients with leukemia (AML-MSC), lung cancer tissues (LC-MSC) and human HeLa cervical cancer cells

$\left(\mathrm{CM}^{\mathrm{HeLa}}\right.$-MSC) were much higher than that in bone marrow-derived MSC (BM-MSC). ${ }^{* *} \mathrm{p}<0.01$ vs. the MSC group, ${ }^{* * *}$ p $<0.001$ vs. the MSC group. (b) The mean fluorescence of the sorted TLR4+ MSC was 34.3, which relatively represents cellular TLR4 expression, while the value of unsorted MSC was only 13.6. (c) The ratio of TLR4+ MSC was enhanced from $13.3 \%$ to $79.6 \%$ after sorting on flow cytometry.

Figure 2. Identification of immunophenotype and differentiation ability of TLR4+ MSC

(a) TLR4+ MSC positively express CD90, CD73, and CD105 and negatively expresses CD45, CD19, CD34, CD11b, and HLA-DR, a finding in accordance with unsorted MSC. (b) TLR4+ MSC maintained the same ability with unsorted MSC to differentiate into osteogenic cells and adipogenic cells.

Figure 3. Cytokine expression of TLR4+ MSC is distinguished from that of MSC

(a) IL-6 and IL-8 were secreted at lower levels by TLR4+ MSC compared with MSC, whereas MCP-1 was expressed at much higher concentrations in the culture supernatant of TLR4+ MSC. ${ }^{* *} \mathrm{p}<0.01$ vs. the MSC group, ${ }^{* * *} \mathrm{p}<0.001$ vs. the MSC group. (b) Due to the enrichment of TLR4 expression, the LPS-induced secretion enhancement of IL-6 and IL-8 was much more significant in TLR4+ MSC than in MSC.

Figure 4. TLR4+ MSC inhibit NK cell proliferation, NKG2D expression and NK cell cytotoxicity

(a) NK cell proliferation was significantly suppressed by TLR4+ MSC or TLR4 activated TLR4+ MSC in vitro. (b) The percentage of NK cells in mouse PBMC was decreased in TLR4+ MSC-treated mice compared with MSC-treated mice. (c) On flow cytometry assay, the expression NKG2D receptor on NK cells was decreased by TLR4+ MSC. (d) The cytotoxicity of NK cells to K562 cells was suppressed by TLR4+ MSC or TLR4 activated TLR4+ MSC. *p $<0.05$, **p $<0.01$, $* * * \mathrm{p}<0.001$. 


\section{Figure 1}

a

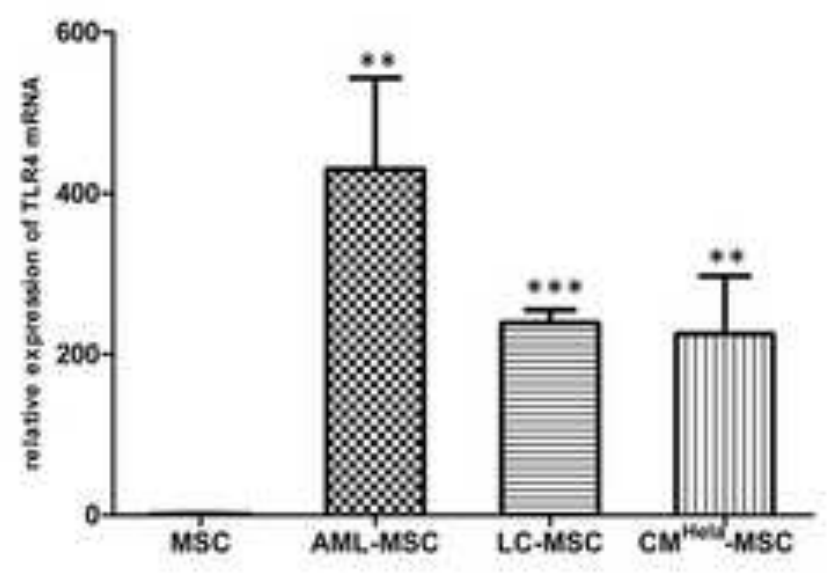

b

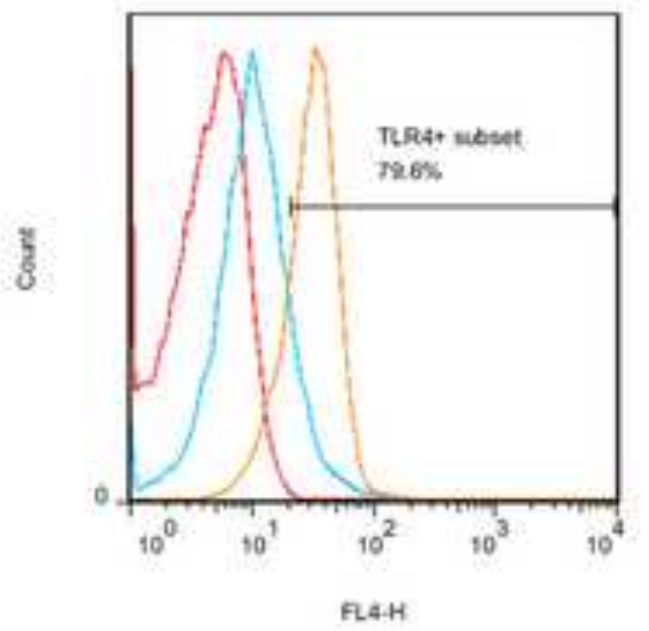

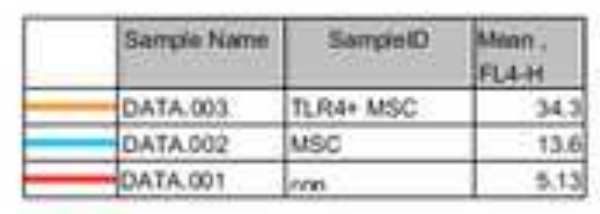

C
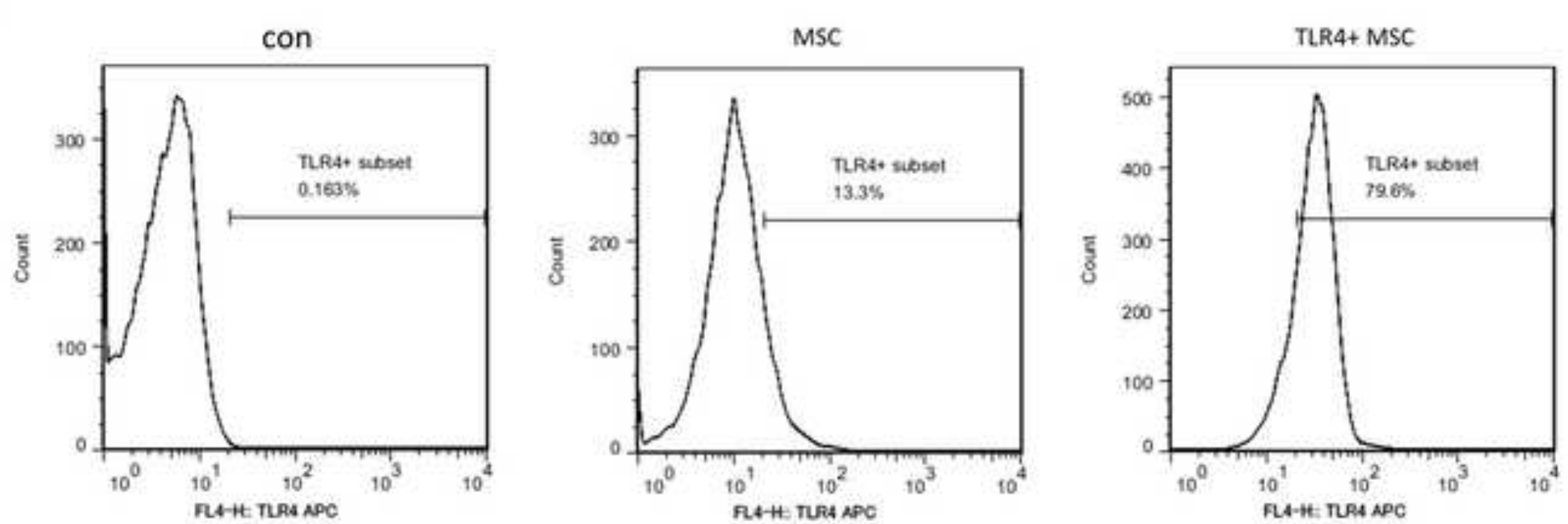
a
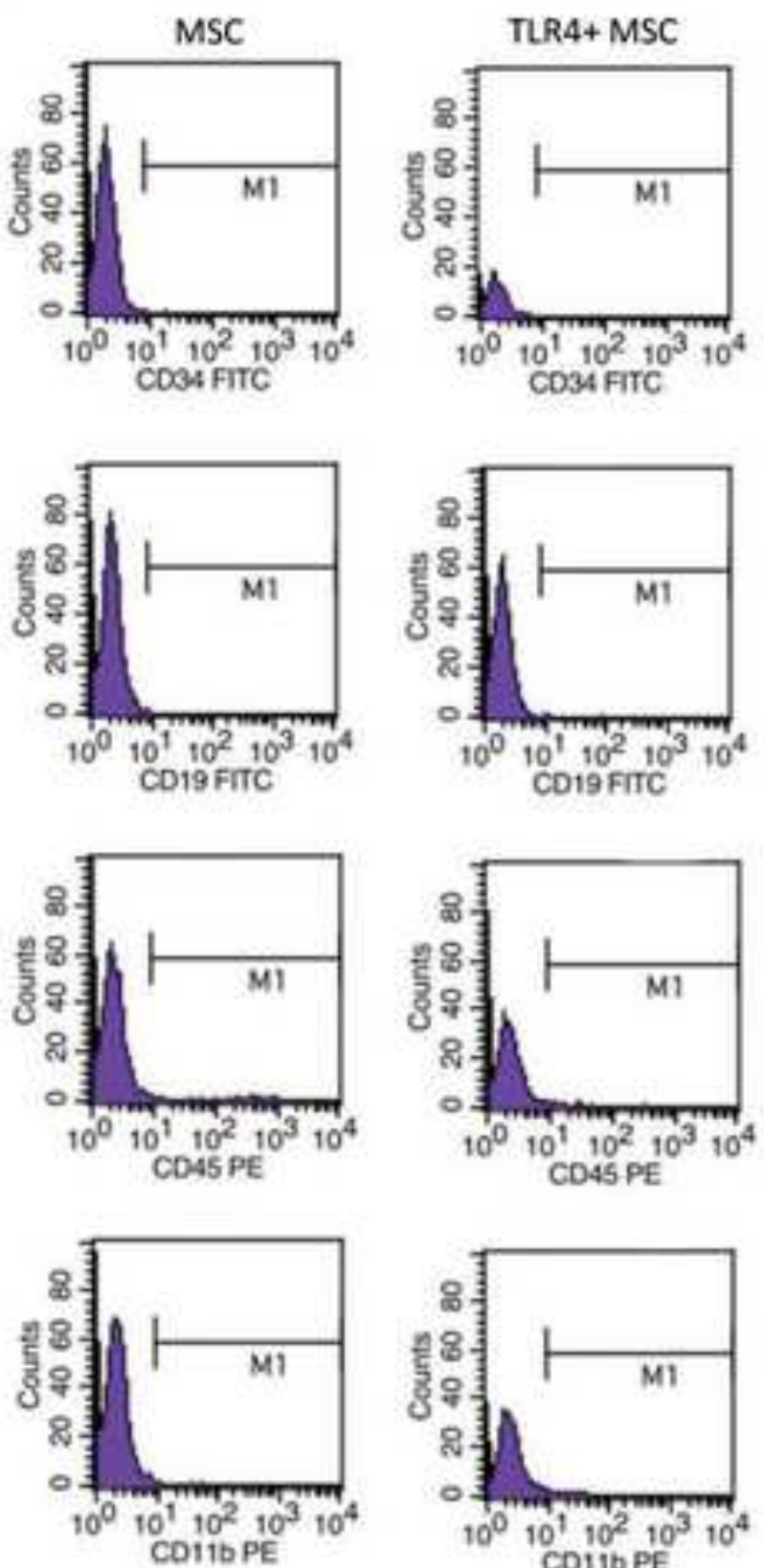

b

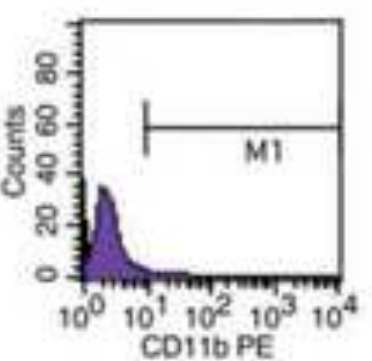

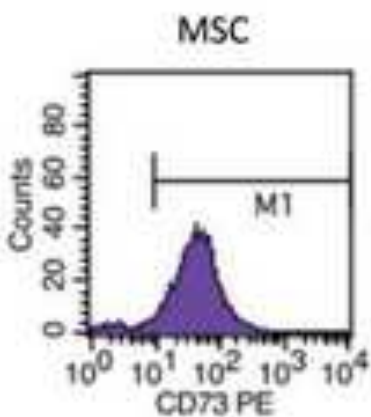
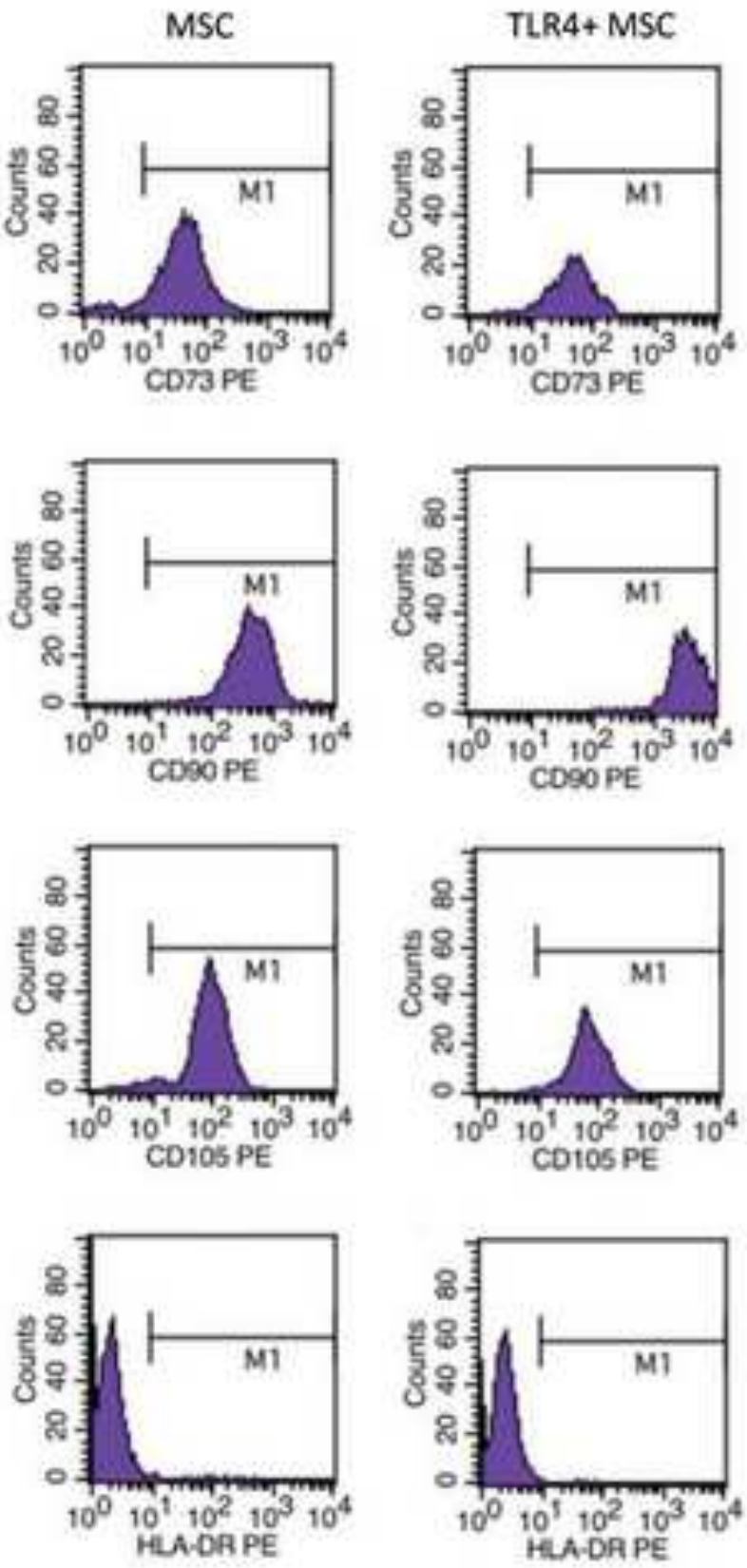
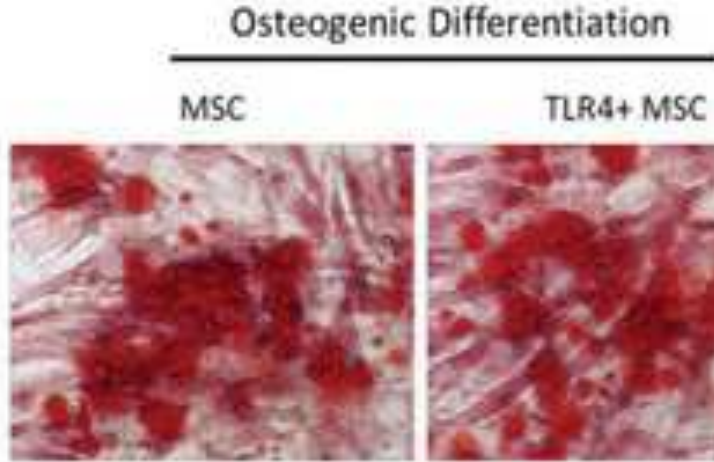

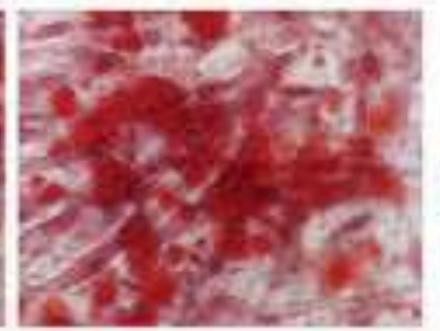

\section{Adipogenic Differentiation}

MSC

TLR4+ MSC

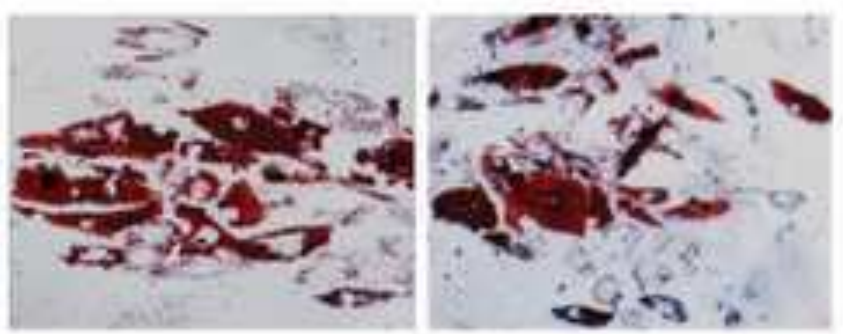


a

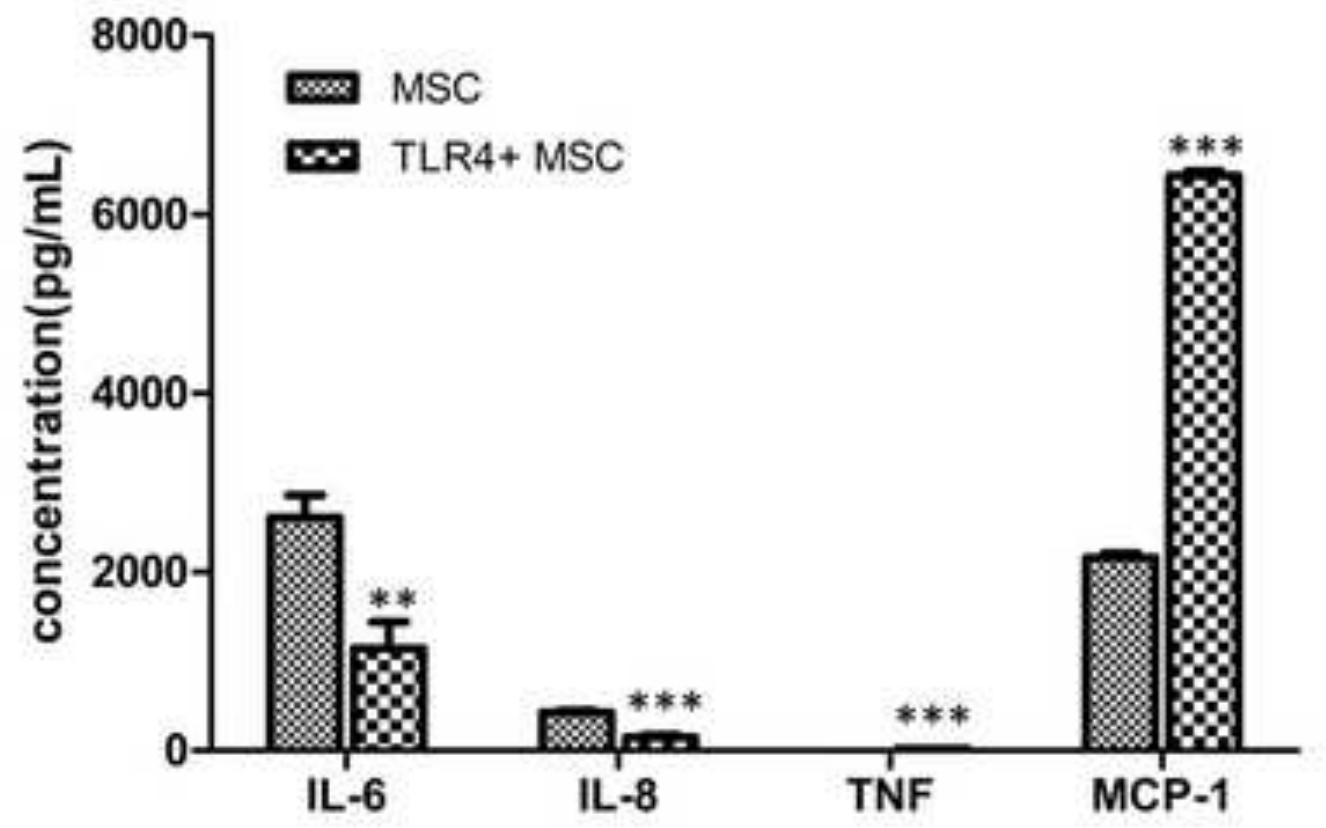

b
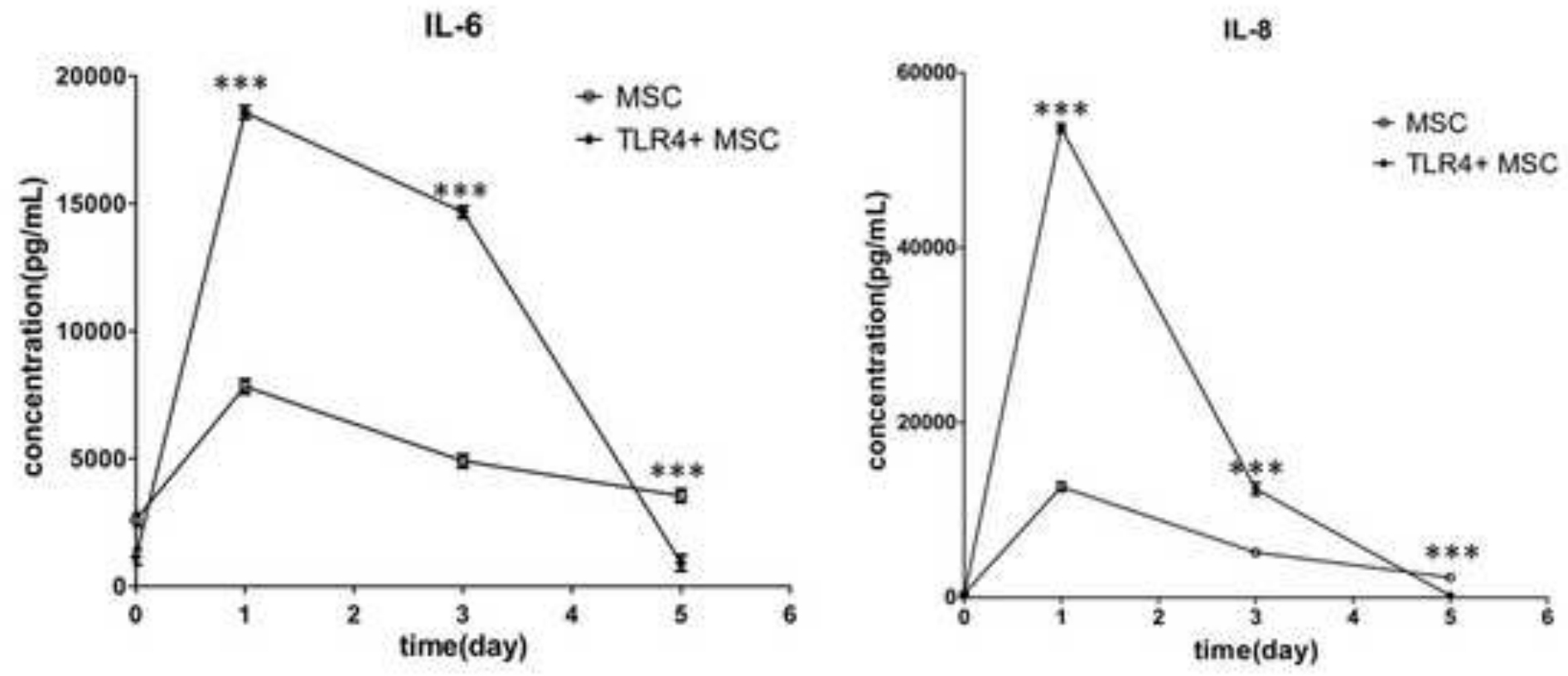
a

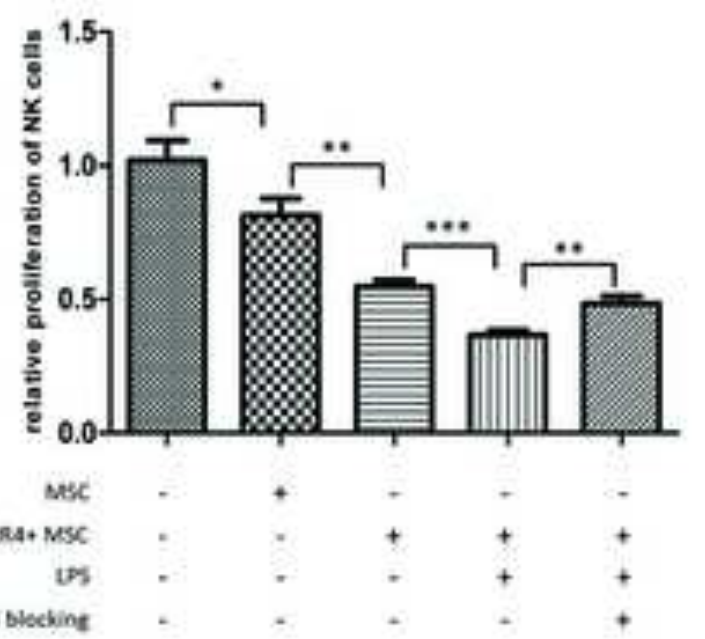

C

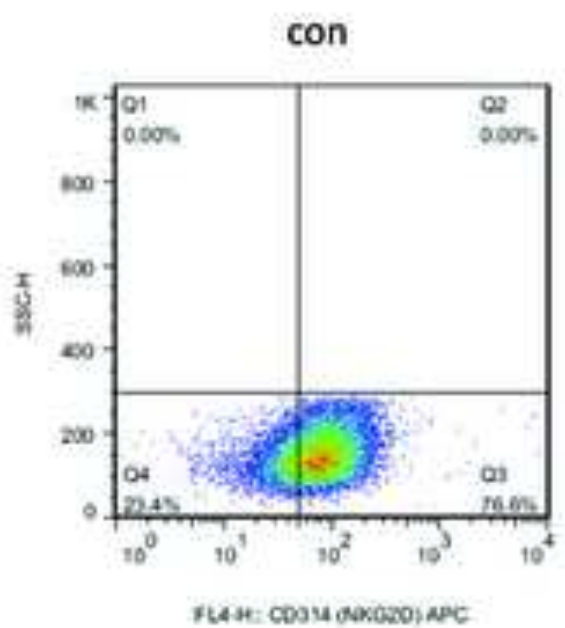

b
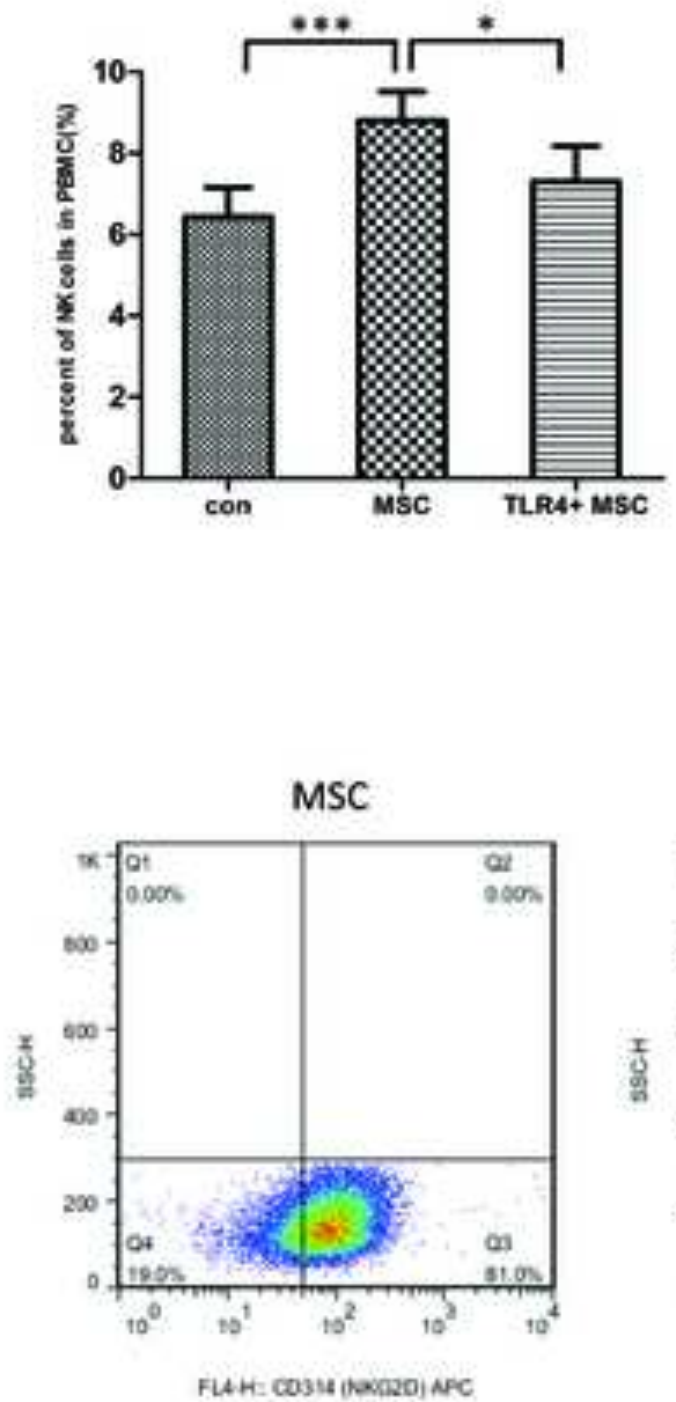

d
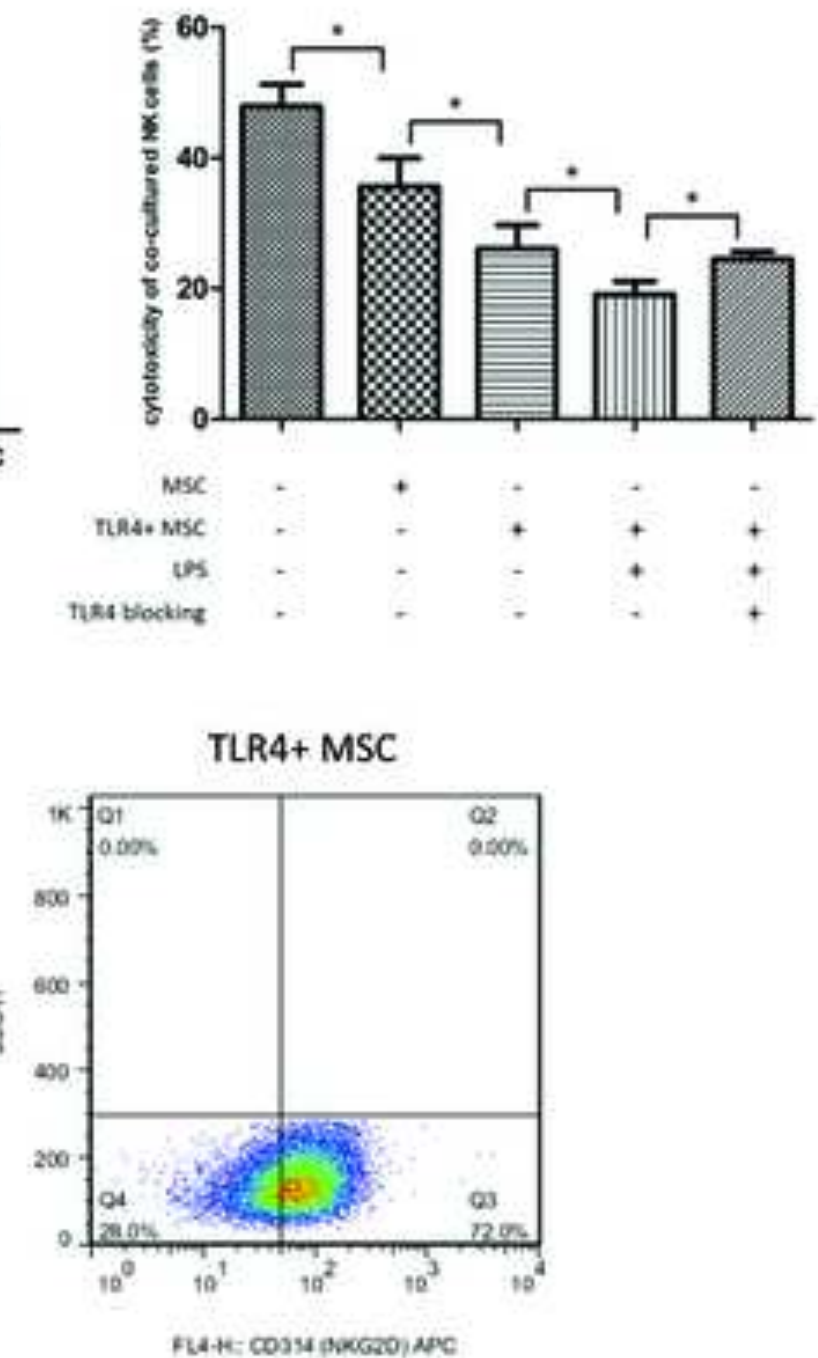